\title{
Imaging characteristics of adult onset Still's disease demonstrated with ${ }^{18}$ F-FDG PET/CT
}

\author{
LEI JIANG ${ }^{1-3}$, YAN XIU ${ }^{1-3}$, TAOYING GU ${ }^{1-3}$, CAIHONG DONG ${ }^{3,4}$, BING WU ${ }^{1-3}$ and HONGCHENG SHI ${ }^{1-3}$ \\ ${ }^{1}$ Department of Nuclear Medicine, Zhongshan Hospital, Fudan University; ${ }^{2}$ Nuclear Medicine Institute \\ of Fudan University; ${ }^{3}$ Shanghai Institute of Medical Imaging; ${ }^{4}$ Department of Ultrasound, \\ Zhongshan Hospital, Fudan University, Shanghai 200032, P.R. China
}

Received August 29, 2016; Accepted May 26, 2017

DOI: $10.3892 / \mathrm{mmr} .2017 .7022$

\begin{abstract}
The diagnosis of adult onset Still's disease (AOSD) is non-specific, and requires the exclusion of other diseases including infectious, inflammatory and malignant diseases. The current study aimed to summarize the imaging characteristics of fluorodeoxyglucose $\left({ }^{18} \mathrm{~F}-\mathrm{FDG}\right)$ positron emission tomography (PET)/computerized tomography (CT) in patients with AOSD. The ${ }^{18} \mathrm{~F}-\mathrm{FDG} \mathrm{PET} / \mathrm{CT}$ characteristic observations of 32 patients with definite AOSD were retrospectively reviewed based on visual interpretation and the semi-quantitative index of standard uptake value of maximum (SUVmax). Among 32 patients, no normal case was observed. Abnormal FDG accumulation by the spleen, bone marrow and lymph nodes was the main observation of the PET/CT images. Totals of $27(84.4 \%)$ and 26 cases $(81.3 \%)$ were identified with diffusely elevated FDG uptake by the spleen and bone marrow, respectively, and the average SUVmax was $4.2 \pm 1.1$ and $4.6 \pm 0.6$, respectively. A total of 20 cases $(62.5 \%)$ showed lymphadenopathy with FDG uptake, with the range of SUVmax from 2.2-13.9. In addition, 7 patients $(21.9 \%)$ were observed to exhibit effusion without FDG uptake, 1 case presented with abnormal FDG uptake by the skin, and another by the right shoulder joint. In addition, no abnormally elevated FDG uptake by the liver or large vessels was observed. Due to non-specific imaging features, ${ }^{18} \mathrm{~F}-\mathrm{FDG} \mathrm{PET} / \mathrm{CT}$ could not be directly helpful in diagnosing AOSD. However, ${ }^{18} \mathrm{~F}-\mathrm{FDG}$ $\mathrm{PET} / \mathrm{CT}$ serves important roles in evaluating the involved extent of AOSD, and guiding the biopsy of lymph nodes, bone marrow or other tissues, which may aid in the development of novel clinical management strategies.
\end{abstract}

Correspondence to: Dr Hongcheng Shi, Department of Nuclear Medicine, Zhongshan Hospital, Fudan University, 180 Fenglin Road, Shanghai 200032, P.R. China

E-mail: shi.hongcheng@zs-hospital.sh.cn

Key words: adult onset Still's disease, fluorodeoxyglucose, positron emission tomography/computerized tomography

\section{Introduction}

Adult onset Still's disease (AOSD) is a rare multi-systemic inflammatory disease of unknown etiology and pathogenesis, which predominantly affects young adults, and most commonly occurs before the age of 35 years old (1-4). Its main features are high spiking fever, evanescent rash, lymphadenopathy, hepatosplenomegaly, leukocytosis, elevated liver enzymes, erythrocyte sedimentation rate, and serum ferritin. However, due to non-specific clinical performance, laboratory and imaging examinations, the diagnosis of AOSD first requires the exclusion of other diseases including infectious, inflammatory and malignant diseases (5-7). Thus, it is important to rule out a wide range of other diseases, including malignant, infectious and rheumatic diseases before the definite diagnosis of $\operatorname{AOSD}(2,6,8)$.

Numerous clinical studies have demonstrated that the important roles of ${ }^{18} \mathrm{~F}$-fluorodeoxyglucose positron emission tomography/computed tomography $\left({ }^{18} \mathrm{~F}-\mathrm{FDG}\right.$ PET/CT) examinations in obtaining differential diagnoses of malignant vs. benign diseases. In addition, ${ }^{18} \mathrm{~F}-\mathrm{FDG}$ PET/CT scanning is more effective in evaluating the involved extent of the disease. At present, the majority of knowledge concerning ${ }^{18} \mathrm{~F}-\mathrm{FDG}$ PET/CT imaging in patients with AOSD is limited to sporadic case reports $(2,9-15)$ and few previous studies $(16,17)$. Thus, the purpose of the present study was to summarize the imaging characteristics of ${ }^{18} \mathrm{~F}-\mathrm{FDG}$ PET/CT and evaluate the potential efficacy of using PET/CT in patients with AOSD.

\section{Materials and methods}

Patients. Between November 2010 and March 2015, 32 patients with definite AOSD were enrolled from Zhongshan Hospital (Shanghai, China) to this retrospective analysis based on the criteria of Yamaguchi et al (18). It contains 4 major (fever, rash, arthritis and leukocytosis) and 5 minor (sore throat, lymphadenopathy, hepatomegaly/splenomegaly, altered liver function test and negative for antinuclear antibodies and rheumatoid arthritis) criteria. A total of 5 or more criteria with at least 2 major criteria are required to make the diagnosis. All procedures performed involving human participants were in accordance with the ethical standards of the Institutional Review Board of Zhongshan Hospital, Fudan University, and 
with the 1964 Helsinki Declaration and its later amendments or comparable ethical standards. The present study was approved by the Ethics Committee of Zhongshan Hospital, Fudan University (Shanghai, China) and informed consent was obtained from all individual participants included in the study.

${ }^{18}$ F-FDG PET/CT scan. ${ }^{18} \mathrm{~F}-\mathrm{FDG}$ PET/CT scanning was performed on a Discovery VCT 64 System (GE Healthcare Biosciences, Milwaukee, WI, USA) with a $15.7 \mathrm{~cm}$ axial field view. Patients were required to fast for a minimum of $6 \mathrm{~h}$ prior to imaging, and serum glucose levels were maintained under $7.4 \mathrm{mmol} / \mathrm{l}$. PET/CT images were obtained approximately $60 \mathrm{~min}$ subsequent to intravenous administration of 3.7-5.6 MBq of ${ }^{18} \mathrm{~F}-\mathrm{FDG}$ per kilogram of body weight. A total of 6 or 7 bed positions (dependent on the patient's height) from the vertex of skull to middle of the thighs were imaged. PET images were acquired for $2.5 \mathrm{~min}$ per bed position. CT was performed on the same scanner without contrast administration. The CT scan data were collected with $140 \mathrm{kV}, 200 \mathrm{mAs}$ (adjusted by auto $\mathrm{mA}$ ) and a gantry rotation speed of $0.8 \mathrm{sec}$. All CT scans were obtained using 3.75-mm-thick axial slices. Integrated PET and CT images were obtained automatically on Xeleris (GE Healthcare Biosciences) or Advantage workstations (GE Healthcare Biosciences).

Visual and semi-quantitative analysis. PET/CT results were analyzed and interpreted by two experienced Nuclear Medicine physicians who were blind to the patients' clinical information, other conventional imaging findings and the pathology results. Increased ${ }^{18} \mathrm{~F}-\mathrm{FDG}$ uptake by spleen and/or bone marrow was defined as that was greater than liver. This method has been previously used to evaluate spleen and bone marrow uptake in lymphoma and leukemia $(19,20)$. In cases of discrepancy regarding $\mathrm{PET/CT}$ results, a consensus was reached after mutual discussion between two physicians. Areas of focally increased accumulation known to represent physiological ${ }^{18}$ F-FDG uptake, such as brown fat, were excluded. For semi-quantitative analysis, the maximal standardized uptake value (SUVmax) was analyzed as published previously (21). SUVmax was calculated as decay-corrected maximum activity concentration in the lesion divided by administered activity divided by body weight in kilograms. The SUVmax of a region of interest located in the right lobe of the liver served as the reference. Associated results, including splenomegaly, lymph node swelling and other lesions, were additionally evaluated. In cases of multiple lymph nodes, SUVmax was calculated for all lesions, and the highest SUVmax was selected.

Pathology. Pathological examinations were performed: 26 cases on bone marrow, and 9 cases on lymph node biopsies. The pathology was reviewed by 2 experienced board-certified pathologists and all cases were clinically monitored a minimum of 1 year.

Statistical analysis. All data are expressed as the means and standard deviations. Statistical analysis was performed using Student's t-test using SPSS software, version 18.0 (SPSS Inc., Chicago, IL, USA). P<0.05 was considered to indicate a statistically significant difference.
Table I. Imaging characteristics of 32 patients with adult onset Still's disease demonstrated with ${ }^{18} \mathrm{~F}-\mathrm{FDG}$ PET/CT.

\begin{tabular}{lcc}
\hline Organs and tissues & Positive cases & $\begin{array}{c}\text { Negative } \\
\text { cases }\end{array}$ \\
\hline Spleen & 27 & 5 \\
SUVmax range & $2.5-6.7$ & $1.5-2.8$ \\
Mean \pm standard deviation & $4.2 \pm 1.1^{\mathrm{a}}$ & $2.1 \pm 0.6^{\mathrm{a}}$ \\
Bone marrow & 26 & 6 \\
SUVmax range & $2.8-7.0$ & $1.3-3.4$ \\
Mean \pm standard deviation & $4.6 \pm 0.6^{\mathrm{a}}$ & $2.6 \pm 0.7^{\mathrm{a}}$ \\
Lymph nodes & 20 & 5 \\
SUVmax range & $2.2-13.9$ & - \\
Mean \pm standard deviation & $5.9 \pm 3.1$ & - \\
Skin & 1 & 31 \\
Joint & 1 & 31 \\
Serous effusion & 7 & 25 \\
\hline
\end{tabular}

${ }^{\mathrm{a}} \mathrm{P}<0.05$.

\section{Results}

Clinical data. A total of 32 patients with AOSD, comprising 25 female and 7 male patients with an average age of $39 \pm 15$ years (range, $19-80$ years) took part in the present study. The patients were admitted to the hospital with various non-specific clinical features, including fever, rash, joint pain, sore throat, fatigue, skin itching, and lymphadenopathy. Fever $(32 / 32,100.0 \%)$ with the range of the maximum temperature (Tmax) from $39-41^{\circ} \mathrm{C}$, rash $(18 / 32,56.3 \%)$ and joint pain $(16 / 32,50.0 \%)$ were the top three clinical symptoms.

${ }^{18}$ F-FDG PET/CT imaging. As presented in Table I, among 32 patients, no normal case was observed. Diffusely abnormal FDG accumulation by the spleen and bone marrow was the main observation on PET/CT images, followed by lymphadenopathy with abnormal FDG uptake. A total of 27 cases out of the 32 patients were identified with diffusely elevated FDG uptake by the spleen, and the average SUVmax was 4.2 \pm 1.1 (range, 2.5-6.7), and 19 of 27 cases exhibited swelling of the spleen. The remaining 5 cases were without abnormal FDG uptake, and only two patients were identified to present with marginal spleen swelling. The mean SUVmax of these 5 spleens was $2.1 \pm 0.6$ (range, $1.5-2.8$ ), which was significantly lower than that of 27 cases $(\mathrm{P}<0.05)$.

In addition, 26 of 32 patients presented with diffuse and homogeneous glucose accumulation by bone marrow of whole body, with the mean SUVmax of 4.6 \pm 0.6 (range, 2.8-7.0). The remaining 6 cases were observed without FDG uptake, and the mean SUVmax was 2.6 \pm 0.7 (range, 1.3-3.4). There was significant difference between the abovementioned two groups $(\mathrm{P}<0.05)$.

Among 32 cases, 20 patients were identified with lymphadenopathy with positive FDG uptake on PET/CT images. The performance of FDG metabolism by lymph nodes was mixed, with an SUVmax range from 2.2-13.9, and the mean 
A

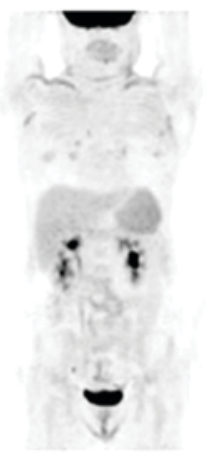

B

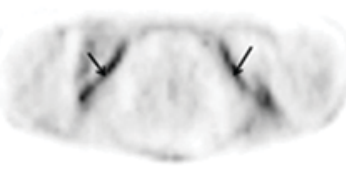

E

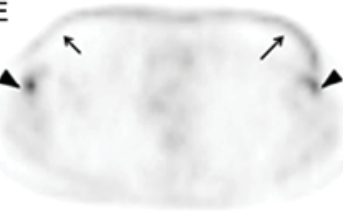

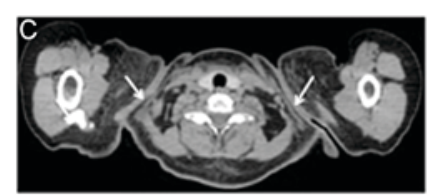
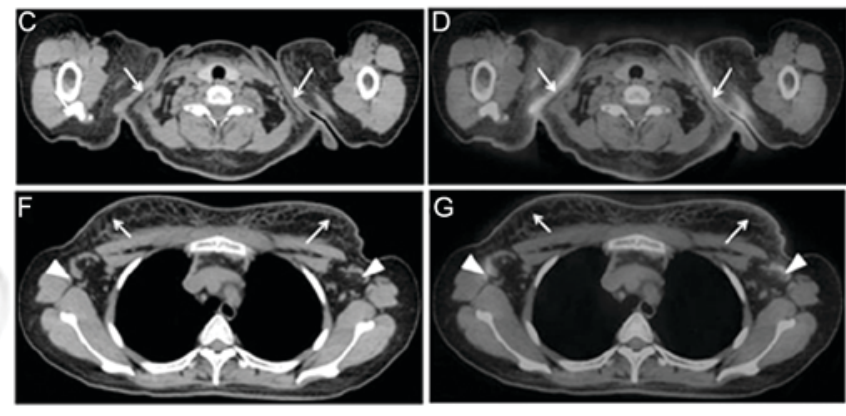

Figure 1. A 36-year-old female patient presented with intermittent fever (Tmax of $\left.39.6^{\circ} \mathrm{C}\right)$ and rash for almost 1 year. AOSD was diagnosed 10 months ago, and the patient received the therapy of CSs. The rash was worsened after the reduction of CSs, and was widely located in the skin of trunk and limbs. ${ }^{18} \mathrm{~F}-\mathrm{FDG}$ PET/CT images are presented: (A) MIP, (B) PET, (C) CT, (D) PET/CT, (E) PET, (F) CT, (G) PET/CT. These images indicated diffusely increased FDG uptake by the skin of the neck, shoulders and chest (arrows), with an SUVmax of 3.1. In addition, marginal swelling of the spleen was observed with diffuse FDG uptake. Lymph nodes of the left cervical, bilateral axillary (triangle arrows), lung hilar and abdominal regions were enlarged with mildly increased radioactivity, of which the SUVmax was 2.2. AOSD, adult onset Still's disease; CSs, corticosteroids; ${ }^{18} \mathrm{~F}-\mathrm{FDG}$, fluorodeoxyglucose; PET, positron emission tomography; CT, computerized tomography; MIP, maximum intensity projection; SUV, standardized uptake value.

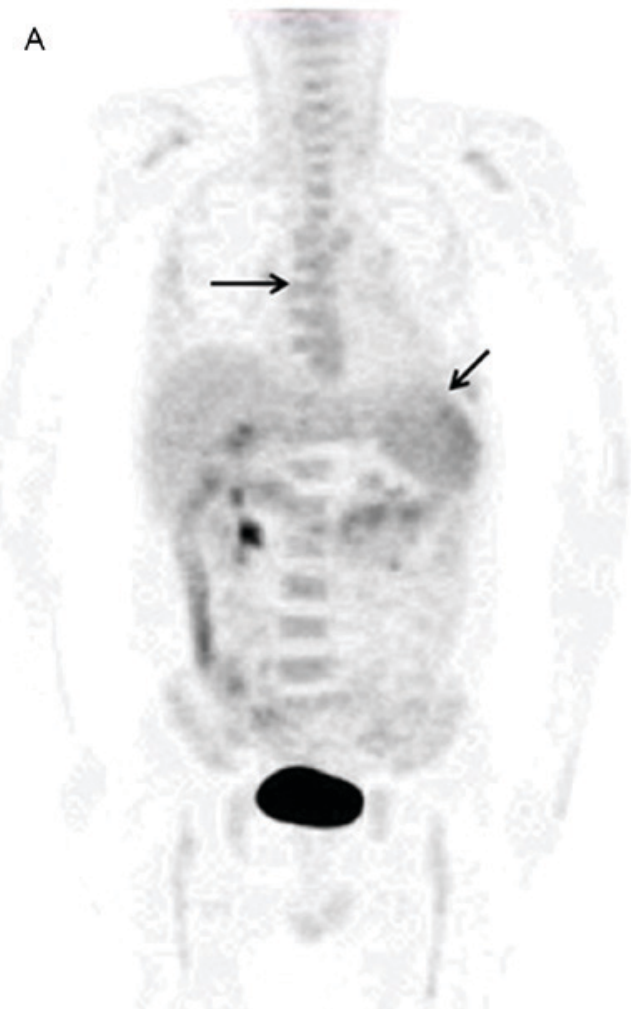

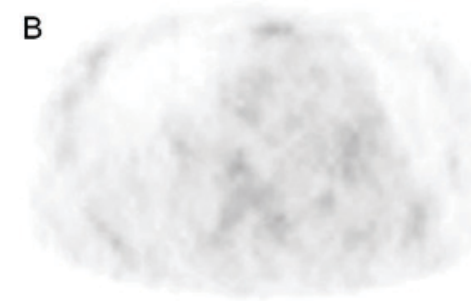
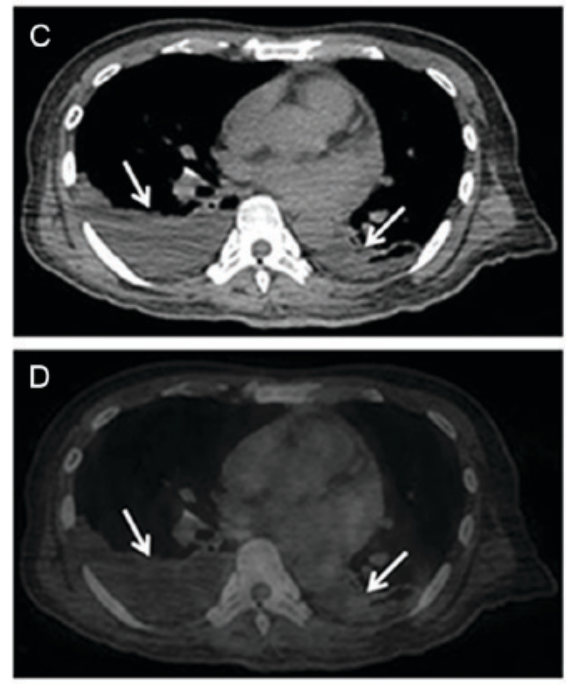

Figure 2. A 57 -year-old male patient presented with fever (Tmax of $\left.39.0^{\circ} \mathrm{C}\right)$ for 3 weeks, which was not relieved by anti-inflammatory therapy. ${ }^{18} \mathrm{~F}-\mathrm{FDG}$ PET/CT imaging indicated that in addition to diffuse FDG uptake by the spleen and bone marrow (A: MIP, black arrows), bilateral pleural effusion with partial atelectasis was observed (B-D: PET, CT and PET/CT, white arrows). ${ }^{18} \mathrm{~F}-\mathrm{FDG}$, fluorodeoxyglucose; PET, positron emission tomography; CT, computerized tomography; MIP, maximum intensity projection.

SUVmax was $5.9 \pm 3.1$. The range of maximum diameter of lymph nodes was from 9.3-24.2 $\mathrm{mm}$, and the mean diameter was 15.3-4.2 $\mathrm{mm}$. The lymph node swelling in 2 cases was limited to local lymph nodes (one in mediastinum, the other in bilateral necks), and the remaining 18 cases displayed varied lymph node involvement (neck, clavicle, axillary, mediastinum, lung hilar, abdominal, retroperitoneal, pelvic and inguinal regions). The most involved lymph nodes were located in the neck (19/32) and chest (18/32), the abdomen and pelvic regions were secondary (13/32).
In addition, 1 case presented with marginally increased FDG uptake by the skin of the neck, shoulders and chest, which was caused by the skin rash of the corresponding part (Fig. 1). Another case displayed elevated FDG activity in the right shoulder joint, but the patient had no history of joint diseases. A total of 7 cases presented with effusion on the PET/CT images, including 4 cases with diffuse effusion (pleural, pericardial, abdominal and pelvic effusion; Fig. 2) and 3 cases with local effusion ( 2 patients with pericardial effusion and 1 patient with pelvic effusion). However, in the present study, no case of liver 
A

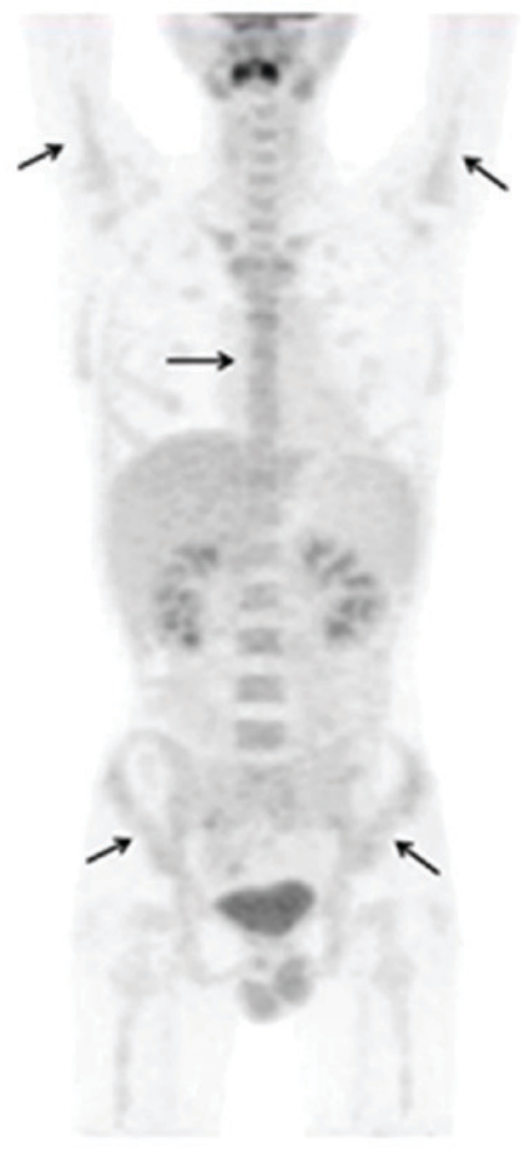

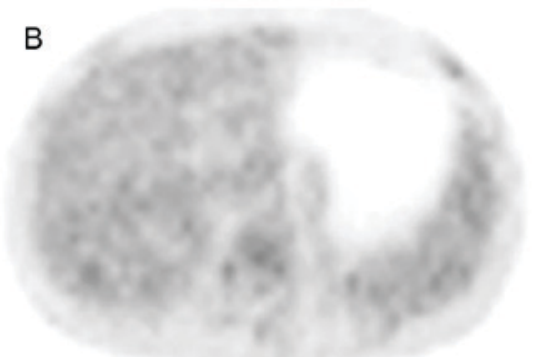
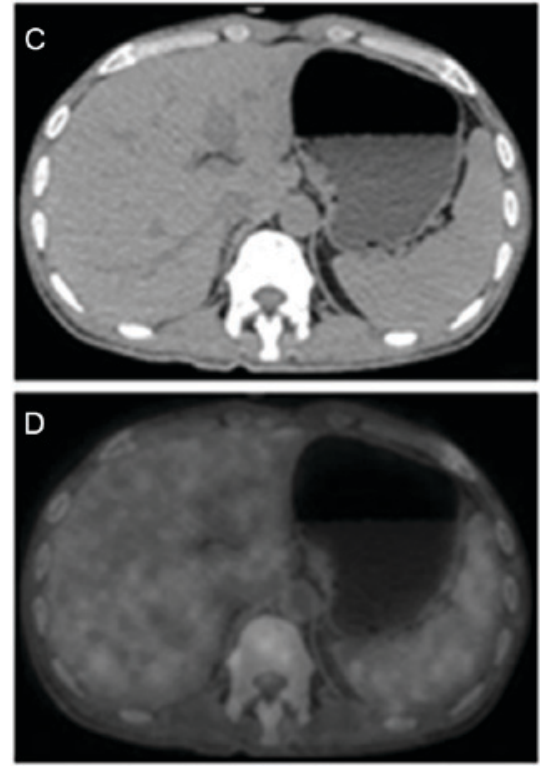

Figure 3. A 28 -year-old male patient presented with fever (Tmax of $41.1^{\circ} \mathrm{C}$ ) and joint pain for 1 month. ${ }^{18} \mathrm{~F}-\mathrm{FDG}$ PET/CT imaging indicated only diffuse increases in FDG accumulation by bone marrow (A: MIP, arrows), of which the SUVmax was 2.8. In addition, marginal spleen swelling without abnormal FDG uptake was also observed (B-D: PET, CT and PET/CT). ${ }^{18}$ F-FDG, fluorodeoxyglucose; PET, positron emission tomography; CT, computerized tomography; MIP, maximum intensity projection; SUV, standardized uptake value.

swelling and/or abnormal glucose metabolism was observed, and the mean SUVmax of the liver was $2.5 \pm 0.5$ (range, 1.7-3.5).

Pathology and clinical follow-up. Among 32 patients, 26 cases underwent bone marrow biopsy: 9 cases displayed normal bone marrow biopsies, 15 cases showed the non-specific reactive hyperplasia of hematopoietic cells, 1 case presented with non-specific low bone marrow hyperplasia, and 1 case with non-specific increased granulocyte ratio. Lymph node biopsies were conducted in 9 patients out of 32, and the results demonstrated non-specific inflammation in 8 cases, and a normal result in 1 case. With the exception of 1 case, the remaining 31 patients responded well to corticosteroids (CSs) and the symptoms were markedly improved. The symptom of this case returned, and was finally improved under the control of methotrexate and methylprednisolone.

\section{Discussion}

AOSD is a chronic systemic inflammatory disease, first described by Bywaters in 1971 (10,22). Due to the non-specific features in clinical performance, laboratory tests and imaging modalities, AOSD remains difficult to diagnose. In the present study, all 32 patients were admitted to the hospital based on fever of unknown origin. In order to exclude malignancy, ${ }^{18} \mathrm{~F}-\mathrm{FDG} \mathrm{PET} / \mathrm{CT}$ scans were performed, and the imaging characteristics of AOSD on PET/CT imaging were summarized and analyzed.

Previous studies have indicated that the results of ${ }^{18} \mathrm{~F}-\mathrm{FDG}$ PET/CT for AOSD are mixed.Funauchi et al (10) presented a case of AOSD with increased FDG activity in the liver and spleen alone. Roy et al (9) reported elevated FDG level in the joints of whole body associated with AOSD in a 28-year-old patient. In another case reported by de Graaff et al (14), increased FDG accumulation by the carotids, the wrist and the large vessels of the legs was demonstrated. Yamashita et al (17) reported that in 7 cases with AOSD, FDG uptake was additionally positive in the pericardium, pleura, salivary glands, eyelids and muscles. Other cases reported increased FDG uptake was identified in multiple lymph nodes across the whole body $(2,12,16,17)$, which could not lead to differential diagnosis with malignant lymphoma. In agreement with previous studies, the ${ }^{18} \mathrm{~F}-\mathrm{FDG}$ PET/CT images in patients with AOSD presented with varied results. Increased FDG activity in the spleen (84.4\%), bone marrow (81.3\%) and lymphadenopathy $(62.5 \%)$ were the most common observations. In addition, $21.9 \%$ of patients were exhibited with effusion without FDG uptake, and another 2 cases showed abnormal FDG uptake by the skin and joint, respectively. Notably, no elevated FDG uptake in the liver and vessels were observed in the present study.

Based on the imaging features of this study, the roles of ${ }^{18}$ F-FDG PET/CT in AOSD were retrospectively investigated. 


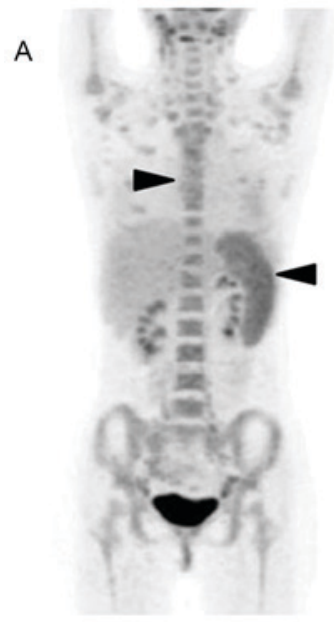

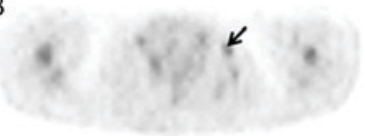
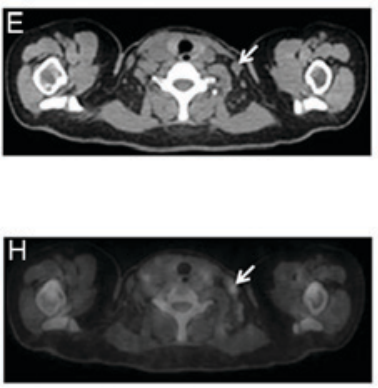

C
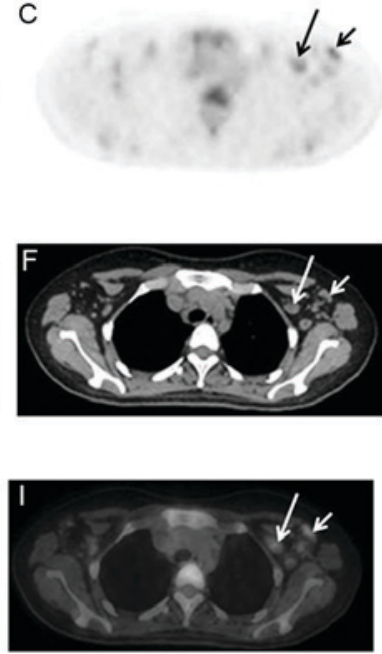

D
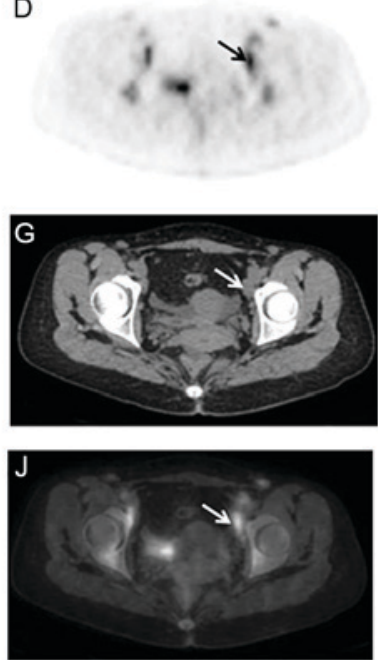

Figure 4. A 28 -year-old female presented with intermittent fever, bilateral knee joints pain, and rash for 1 month, and Tmax was $40.4^{\circ} \mathrm{C}$. The treatment of antibiotics was invalid, and local CT displayed multiple enlarged lymph nodes of cervical and axillary regions. ${ }^{18} \mathrm{~F}-\mathrm{FDG}$ PET/CT scan indicated that the spleen was markedly enlarged with FDG accumulation, with SUVmax of 5.2, and diffuse radioactivity uptake was observed in the bone marrow with an SUVmax of 5.0 (A: MIP, triangle arrows). In addition, multiple lymph nodes of cervical, supraclavicle, mediastina, axillary, abdominal, retroperitoneal, pelvic and inguinal regions were enlarged with increased radioactivity (B-D: PET, E-G: CT, and H-J: PET/CT, arrows). CT, computerized tomography; ${ }^{18}$ F-FDG, fluorodeoxyglucose; PET, positron emission tomography; SUV, standardized uptake value; MIP, maximum intensity projection.

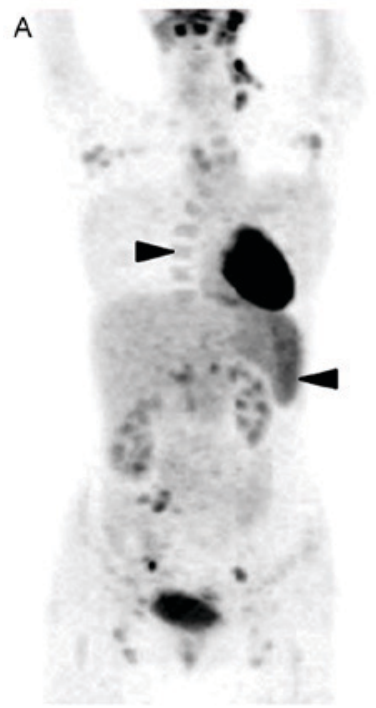

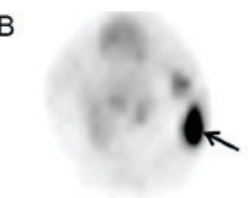
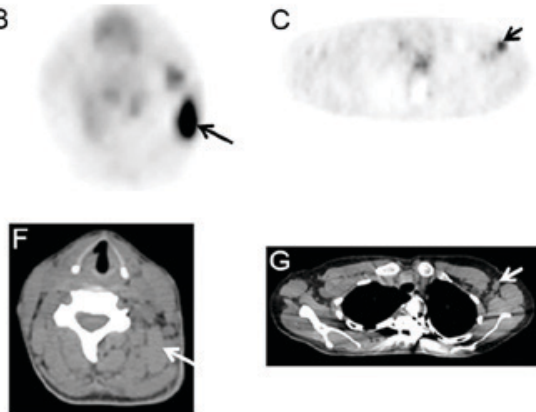

D
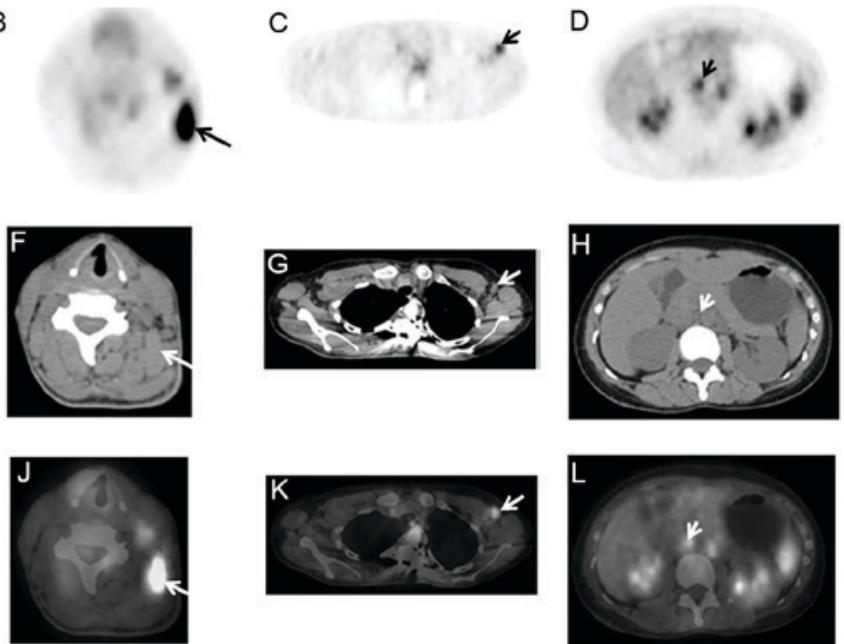
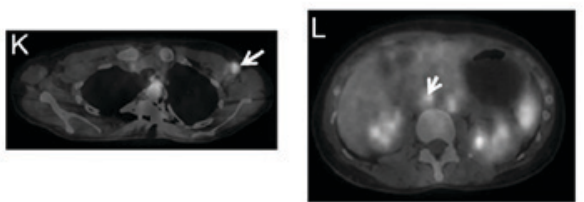

E
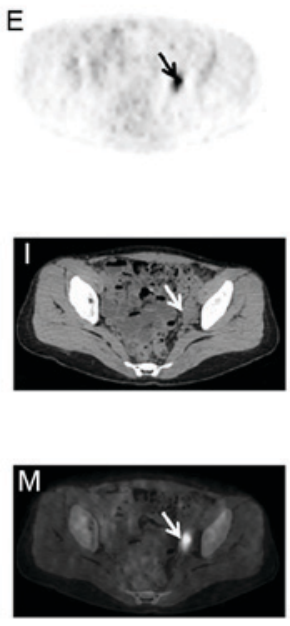

Figure 5. A 25-year-old female presented with a mass located in the left cervical region for 1 month, fever (Tmax of $\left.40.0^{\circ} \mathrm{C}\right)$ and joint pain for 3 weeks. The patient had history of corrective operation for deformities of the spine 10 years ago. ${ }^{18} \mathrm{~F}-\mathrm{FDG}$ PET/CT imaging displayed that in addition to diffuse FDG uptake by the spleen and bone marrow (A: MIP, triangle arrows), multiple lymphadenopathy with abnormal FDG uptake (arrows) was observed (B-E, PET; F-I, CT; and J-M, PET/CT). The biopsy of left cervical lymph node (B, F and G; arrows) was taken and the pathology was non-specific inflammation. The biopsy of bone marrow was non-specific reactive hyperplasia of hematopoietic cells. After the therapy of CSs for 1 month, ultrasound displayed that the lymph nodes of left neck and axillary obviously shrine. ${ }^{18} \mathrm{~F}-\mathrm{FDG}$, fluorodeoxyglucose; PET, positron emission tomography; CT, computerized tomography; MIP, maximum intensity projection.

Firstly, ${ }^{18} \mathrm{~F}-\mathrm{FDG}$ PET/CT could display both anatomic and functional changes of AOSD, and evaluated the involved extent of AOSD. For example, as presented in Fig. 3, the case only displayed diffusely elevated FDG activity in bone marrow of whole body on PET/CT images. The case in Fig. 4 demonstrated that although multiple enlarged lymph nodes of the whole body with mildly increased radioactivity were displayed on PET/CT images, the benign diseases of lymph nodes such as inflammation rather than malignancies were diagnosed based on the size, morphology, density, and SUVmax of the lymph nodes in this case. However, for other cases, as presented in Figs. 5 and 6 , which was difficult to make differential diagnosis with lymphoma. Secondly, it could guide the biopsy of tissues with abnormal FDG accumulation including lymph nodes, bone marrow, skin and other tissues. Regarding the cases in Figs. 5 and 6 , the biopsies of the lymph nodes were performed under the guide of ${ }^{18} \mathrm{~F}-\mathrm{FDG}$ PET/CT imaging, which contributed to suggest the proper lymph node applied for the biopsy. Finally, 

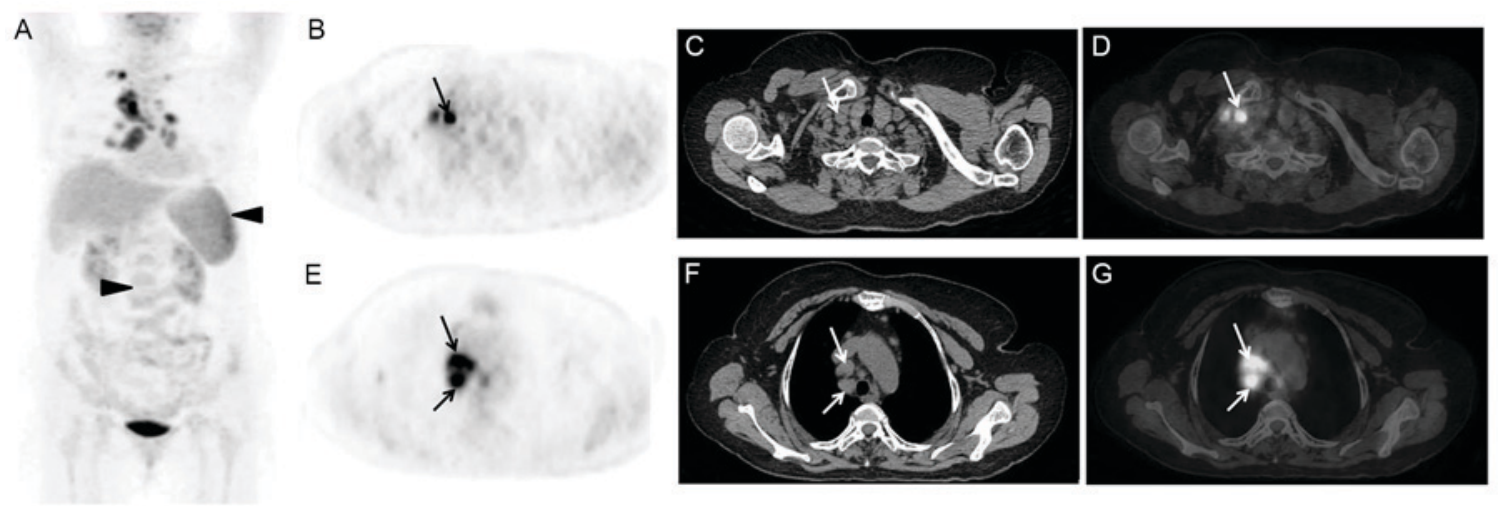

Figure 6. A 67-year-old female presented with fever (Tmax of $39.0^{\circ} \mathrm{C}$ ) for 4 weeks, and anti-inflammatory therapy was invalid. ${ }^{18} \mathrm{~F}-\mathrm{FDG}$ PET/CT imaging observed that diffuse FDG uptake by the spleen and bone marrow (A: MIP, triangle arrows), and multiple lymphadenopathy with abnormal FDG uptake in the cervical, supraclavicle, mediastinum and lung hilar regions (B and E, PET; C and F, CT; D and G, PET/CT; arrows). The pathology after the biopsy of the right supraclavicle lymph node (B-D; arrows) led to the diagnosis of non-specific inflammation. The bone marrow biopsy indicated non-specific and mild hyperplasia of hematopoietic cells, predominantly myeloid, and lymphocytes and plasma cells within normal range. During the follow-up, the lymph nodes of supraclavicle, mediastinum and lung hilar regions were significantly decreased or disappeared on chest CT images. ${ }^{18} \mathrm{~F}-\mathrm{FDG}$, fluorodeoxyglucose; PET, positron emission tomography; CT, computerized tomography; MIP, maximum intensity projection.

${ }^{18} \mathrm{~F}-\mathrm{FDG}$ PET/CT is helpful in the post-treatment evaluation of AOSD. Although these data weren't investigated in the present study, Choe et al (2) and Yamashita et al (17) identified significant improvements (decreased SUV level) in the initially abnormally sized radioactive lesions in the follow-up images of PET/CT.

There were several limitations in the present study. i) As a retrospective study, there may have been a substantial selection bias on the basis of the physicians' decisions to request ${ }^{18}$ F-FDG PET/CT examinations; ii) Due to the small size of the population in the present study, the imaging characteristics of AOSD on ${ }^{18} \mathrm{~F}-\mathrm{FDG}$ PET/CT could not be fully investigated; iii) the majority of patients with AOSD respond well to CS treatment, and other imaging modalities including ultrasound and local CT are commonly conducted to monitor AOSD, therefore, there is a lack of data and information concerning the follow-up PET/CT images following the appropriate therapy for AOSD.

In conclusion, due to the non-specific imaging features on ${ }^{18} \mathrm{~F}-\mathrm{FDG}$ PET/CT examinations, ${ }^{18} \mathrm{~F}$-FDG PET/CT scans could not directly aid in the diagnosis of AOSD. The final diagnosis of AOSD is based on clinical performance, laboratory and imaging examinations, and pathology. However, for AOSD, ${ }^{18} \mathrm{~F}$-FDG PET/CT serves important roles in evaluating the involved extent, and guiding the biopsy of the lymph nodes, bone marrow or other tissues, which may aid in the development of novel strategies for clinical management.

\section{Acknowledgements}

The study was in part supported by the National Science Foundation for Scholars of China (grant nos. 81271608 to Dr Hongcheng Shi and 81571703 to Dr Lei Jiang), and funding sponsored by Shanghai Pujiang Program (grant no. 2015PJD006) and Zhongshan Hospital Outstanding Youth Talent Program (grant no. 2015ZSYXQN17). The abstract of the present study was presented and published at the Society of Nuclear Medicine and Molecular Imaging (SNMMI) conference held in San Diego, CA, USA in June, 2016.

\section{References}

1. Efthimiou P, Paik PK and Bielory L: Diagnosis and management of adult onset Still's disease. Ann Rheum Dis 65: 564-572, 2006

2. Choe JY, Chung DS, Park SH, Kwon HH and Kim SK: Clinical significance of ${ }^{18} \mathrm{~F}$-fluoro-dexoxyglucose positron emission tomography in patients with adult-onset Still's disease: Report of two cases and review of literatures. Rheumatol Int 30: 1673-1676, 2010.

3. Senthilvel E, Papadakis A, McNamara M and Adebambo I: Adult-Onset Still Disease (AOSD). J Am Board Fam Med 23: 418-422, 2010

4. Mamun AA, Sutradhar SR, Alam MB and Bhattacharjee M: Management of adult Still's disease-an update. Mymensingh Med J 20: 520-527, 2011.

5. Fautrel B, Zing E, Golmard JL, Le Moel G, Bissery A, Rioux C, Rozenberg S, Piette JC and Bourgeois P: Proposal for a new set of classification criteria for adult-onset still disease. Medicine (Baltimore) 81: 194-200, 2002.

6. Gerfaud-Valentin M, Maucort-Boulch D, Hot A, Iwaz J, Ninet J, Durieu I, Broussolle C and Sève P: Adult-onset still disease: Manifestations, treatment, outcome, and prognostic factors in 57 patients. Medicine (Baltimore) 93: 91-99, 2014.

7. Riera E, Olive A, Narváez J, Holgado S, Santo P, Mateo L, Bianchi MM and Nolla JM: Adult onset Still's disease: Review of 41 cases. Clin Exp Rheumatol 29: 331-336, 2011.

8. Kádár J and Petrovicz E: Adult-onset Still's disease. Best practice \& research. Clin Rheumatol 18: 663-676, 2004.

9. Roy SG, Karunanithi S, Dhull VS, Bal C and Kumar R: ${ }^{18}$ F-FDG PET/CT aids the diagnosis of adult onset Still's disease in a patient with fever of unknown origin. Rev Esp Med Nucl Imagen Mol 33: 392-393, 2014.

10. Funauchi M, Ikoma S, Kishimoto K, Shimazu H, Nozaki Y, Sugiyama M and Kinoshita K: A case of adult onset Still's disease showing marked accumulation in the liver and spleen, on positron emission tomography-CT images. Rheumatol Int 28: 1061-1064, 2008

11. Kato T, Fujii K, Wakabayashi T, Tanaka A and Hidaka Y: A case of cutaneous polyarteritis nodosa manifested by spiking high fever, arthralgia and macular eruption like adult-onset Still's disease. Clin Rheumatol 25: 419-421, 2006.

12. Cai L, Chen Y and Huang Z: Elevated FDG activity in lymph nodes as well as the spleen and liver in a patient with adult-onset still disease. Clin Nucl Med 37: 1009-1010, 2012.

13. Ahn BC, Lee SW and Lee J: Intense accumulation of F-18 FDG in colonic wall in adult onset still disease with pseudomembranous colitis. Clin Nucl Med 33: 806-808, 2008.

14. de Graaff LC, ten Broek MR and Schweitzer DH: Is Still's disease still one disease? A case of Adult-onset Still's disease showing accumulation in the carotids and the large vessels of the legs on positron emission tomography: CT images. Rheumatol Int 32: 2487-2490, 2012 
15. Yazisiz V and Yazisiz H: Comment on: Is Still's disease still one disease? A case of adult-onset Still's disease showing accumulation in the carotids and the large vessels of the legs on positron emission tomography: CT images. Rheumatol Int 33: 1373-1374, 2013.

16. Dong MJ, Wang CQ, Zhao K, Wang GL, Sun ML, Liu ZF and Xu L: ${ }^{18} \mathrm{~F}-\mathrm{FDG}$ PET/CT in patients with adult-onset Still's disease. Clin Rheumatol 34: 2047-2056, 2015.

17. Yamashita H, Kubota K, Takahashi Y, Minamimoto R, Morooka M, Kaneko H, Kano T and Mimori A: Clinical value of ${ }^{18} \mathrm{~F}$-fluoro-dexoxyglucose positron emission tomography/computed tomography in patients with adult-onset Still's disease: A seven-case series and review of the literature. Mod Rheumatol 24: 645-650,2014.

18. Yamaguchi M, Ohta A, Tsunematsu T, Kasukawa R, Mizushima Y, Kashiwagi H, Kashiwazaki S, Tanimoto K, Matsumoto Y, Ota T, et al: Preliminary criteria for classification of adult Still's disease. J Rheumatol 19: 424-430, 1992.
19. Arimoto MK, Nakamoto $Y$, Nakatani K, Ishimori $T$, Yamashita K, Takaori-Kondo A and Togashi K: Increased bone marrow uptake of 18F-FDG in leukemia patients: Preliminary findings. Springerplus 4: 521, 2015.

20. Carr R, Barrington SF, Madan B, O'Doherty MJ, Saunders CA, van der Walt J and Timothy AR: Detection of lymphoma in bone marrow by whole-body positron emission tomography. Blood 91: 3340-3346, 1998

21. Jiang L, Tan H, Panje CM, Yu H, Xiu Y and Shi H: Role of 18F-FDG PET/CT imaging in intrahepatic cholangiocarcinoma. Clin Nucl Med 41: 1-7, 2016.

22. Bywaters EG: Still's disease in the adult. Ann Rheum Dis 30: 121-133, 1971. 